\title{
Simulation of Blood Flow and Nanoparticle Transport in a Stenosed Carotid Bifurcation and Pseudo-Arteriole
}

\author{
Graham Doig ${ }^{1, *}$, Guan H. Yeoh ${ }^{1,2}$, Victoria Timchenko', Gary Rosengarten ${ }^{1}$, \\ Tracie J. Barber ${ }^{1}$, Sherman C.P. Cheung ${ }^{3}$ \\ ${ }^{1}$ School of Mechanical and Manufacturing Engineering, The University of New South \\ Wales, Sydney, NSW 2052, Australia \\ ${ }^{2}$ Australian Nuclear Science and Technology Organisation (ANSTO), PMB 1, Menai, \\ NSW 2234, Australia \\ ${ }^{3}$ School of Aerospace, Mechanical \& Manufacturing Engineering, The Royal Melbourne \\ Institute of Technology, Bundoora, VIC 3083, Australia
}

Received: 21 October 2011, Accepted: 15 February 2012

\begin{abstract}
:
Numerical simulation of flow through a realistic bifurcated carotid artery geometry with a stenosis has been conducted for comparison to experimental measurements. The behaviour of simplified therapeutic nanoparticles in relatively low concentration was observed using a discrete particle approach. The role of size (diameters from $500 \mathrm{~nm}$ to $50 \mathrm{~nm}$ ) in determining particle residence time and the potential for both desirable and undesirable wall interactions was investigated. It was found that mean particle residence time reduced with decreasing particle diameter, and the percentage of particles experiencing one or more wall interactions increased simultaneously. Further simulations were conducted on a scaled-down version of the geometry which approximated the size and flow conditions of an arteriole with capillary branches, and in this instance the mean residence time increased with decreasing particle diameter, owing largely to the greater influence of Brownian motion. $33 \%$ of all $50 \mathrm{~nm}$ particles were involved in wall interactions, indicating that smaller particles would have a greater ability to target, for instance, cancerous tumours in such regions.
\end{abstract}

\section{INTRODUCTION}

The use of therapeutic nanoparticles to attack malignant tumors, whether by highly-targeted delivery of drugs or by thermal ablation of cancerous tissue, is a technique that has been receiving considerable attention in recent years [1], particularly with regards to the role that particle size and shape plays. Compared to laboratory and clinical trials, relatively little numerical work has been conducted on this problem, and thus the potential for simulation to aid in understanding of particle behaviour and design of more effective delivery has been under-exploited. The work presented here represents a preliminary study into the extent to which particle size influences transport through vasculature at both artery and arteriole/capillary scale.

The potential to treat tumors directly and in high dosage without inducing undesirable effects on the rest of the body is being widely investigated in medicine, and may eventually lead to highly effective treatments [1]. However, much of the research to-date presents occasionally-conflicting conclusions about the role of particle size and shape, and raises considerable issues with regards to safety. For instance, damage from localized hyperthermia from ablative treatments involving metallic particles, or toxicity caused by the retention of large concentrations of particles in the spleen, liver and other organs [2]. Toxicity, particularly with regards to silver and gold particles accumulating in the liver, is partially dependent on particle size, thought to be a result of the larger surface area that a set mass of small particles will present [3].

Nevertheless, intravascularly-injected particles have proven useful for the early detection, imaging and therapy of diseases and have the potential to enable on-time diagnosis, molecularlytargeted biomedical imaging and the timely delivery of multiple therapeutic agents $[1,4]$. The general conclusion of clinical trials for particles of varying size is that smaller particles have the best ability to reach their target [5], but an incomplete understanding of the reasons for this persists. 
Beyond the ability to treat a tumour, therapeutic nanoparticles will be required to perform well at multiple duties before optimal treatment can be developed. They must be able to travel relatively freely and efficiently through the bloodstream until the region of interest, such as a tumor. They then are required to target the tumor specifically, such that they will arrive in maximum concentration where they are intended. The complex process of infiltrating the cancerous cells to deliver drugs or perform ablative duties usually necessitate particles of $500 \mathrm{~nm}$ or less penetrating the endothelial cells via fenestrated tissue - this is commonly referred to as the enhanced permeation and retention effect (EPR). Particles continuing to circulate through the body must also eventually be excreted through the kidneys. To perform all these tasks independently, certain sizes and shapes, amongst other attributes, of the particles would be preferable. To perform them all requires certain compromises that make it likely that no single system of specific size, shape, elasticity and targeting properties will suffice for quantitative attack on some targets [6].

For transport of a particle in the body, the diameter will, in part, dictate particle velocity, diffusion, and adhesion to walls in blood vessels, airways and intestines (and thus the potential for phagocytosis). The shape, charge, and chemistry of the particle will influence these properties in more complex ways [7], and mechanical stiffness of the particle is also a considerable factor [8]. After administration of treatment in rodents, small particles $(<30 \mathrm{~nm})$ are eliminated by renal excretion [9]. Larger particles $(150-300 \mathrm{~nm})$ are found mainly in the liver and spleen [10,11], and particles of $30 \mathrm{~nm}-150 \mathrm{~nm}$ typically end up in the heart, kidney and stomach $[12,13]$. While size is therefore clearly influential for both system clearance and biodistribution, it is noted that the parameter is not often well controlled or described in literature [5], often due to issues stemming from measurement techniques.

For targeted applications, the diameter of the particle will affect the degree to which particles fit the contours of target cell membranes. Shear induced by blood flow could detach the particle, and thus the size will determine the longevity of the targeted attachment. Non-specific interactions (van der Waals, electrostatic and steric interactions) with the blood vessel walls are also important to the effectiveness of vascular targeting. "Ideal" size requirements for nanoparticles so far developed for cancer treatment are between 70 and $200 \mathrm{~nm}$ [14].

Decuzzi et al. conducted trials on mice in which particles of spherical, hemispherical, cylindrical and discoidal shape were injected [2]. The number of particles accumulating in the major organs and within the tumour mass was measured using elemental silicon analysis. Smaller spherical particles were found to provide a more uniform tissue distribution, but discoidal particles were observed to accumulate more than others in most organs bar the liver, where cylindrical particles were deposited to a greater extent. The study also indicated that the strength of adhesion of spherical beads to biological substrate reduced with increasing particle diameter, meaning larger particles would be less likely to resist a shear stress dislodgement force.

Doshi et al. state that direct observations of carrier flow in blood vessels are very challenging due to practical issues associated with visualisation of particles in blood vessels (nanoparticles cannot be visualised at an individual level) and isolating contributions from various confounding factors [15]. They addressed this challenge by using their synthetic system, though one can see that accompanying numerical simulation would be very useful. As with many other experimental studies, the hydrodynamic performance of the particles cannot be assessed in detail other than in broad generalisations and inferences from the concentrations and final locations of injected particles. Micro-meter scale particles $(1 \mu \mathrm{m}$ to $20 \mu \mathrm{m})$ were used and can provide advantages over nanoparticles for some therapeutic delivery applications, however they are typically larger than those used for drug delivery. The effect of shape was amplified at larger particle sizes, which it was speculated was due to higher drag or higher settling velocity of larger particles compared to smaller ones, and additionally the channel bifurcation produced significantly different flow and adhesion behaviour than that observed in a simpler linear channel.

"Margination dynamics" is a physiological term conventionally used to describe the lateral drift of platelets from the core blood vessel towards the endothelial walls. Marginating particles can be designed specifically to move preferentially in close proximity to the walls, and this can be highly desirable in vascular targeting [16]. Red blood cells exhibit a behaviour opposite to margination, with cells accumulating preferentially within the core of the vessels, avoiding interaction with the walls. This core accumulation is known as "plasma skimming" and was described by Fahraeus and Lindqvist [17]. Particles designed to marginate tend to accumulate in the cell-free layer near the walls, and Charoenphol et al. concluded that pulsatility in blood flow 
further enhanced the margination of microspheres while having minimal effect on the adhesion of nanospheres [18].

While much of the recent research, as described above, focuses on particle concentrations as a function of size and shape or other parameters, this is very much a cause-and-effect analysis that does not necessarily yield reliable insight into the importance or otherwise of the hydrodynamics of particle transport at any particular point in the circulation. While particle shape has been shown to be an important factor in cell adhesion and the surface area is critical for drug-loaded particles, it can be expected that, particularly in flowfields where particle diameter is insignificant compared to the vasculature in question, the particle trajectory will not be sensitive to this parameter. Therefore, this paper concentrates on a preliminary investigation into the role of particle size on the particle dynamics in a patient-specific geometry. The primary advantage of this modeling is that the particle size and concentration is perfectly controlled, thus removing some of the uncertainty which can arise even from well-designed experiments.

In order to examine numerically the transport of nanoparticles in a in a realistic setting, with a simplified model for particle trajectories, a bifurcated carotid artery geometry derived from highresolution magnetic resonance imaging was used following the study of Cheung et al. [19]. One arm of the bifurcation (the internal carotid artery) features a stenosis as indicated in figure 1 , which provides the flowfield with a point of constriction and high shear stress, and therefore high local flow velocity.

The work of Cheung et al. was used as a means of benchmarking the flow solution prior to the introduction of particles into the simulation. Their experimental reproduction of the stenosed bifurcated artery was scaled up by a factor of ten from the original geometry, in order to allow a clear silicone rig of reasonable size to be built for measurements made using particle image velocimetry (PIV). From this point on, any reference to the artery refers to this enlarged scale. The Reynolds number was kept consistent with that which could be expected at the original scale by changing the effective viscosity of the working fluid (kept at a constant $25^{\circ} \mathrm{C}$ ), and the flow was steady (non-pulsatile). For more details on the reference experiments, the reader is directed to Cheung et al. [19] - it is sufficient here for the sake of brevity to state that all known aspects of the experiment have been reproduced numerically as faithfully as possible, with pertinent attributes and boundary conditions outlined in table 1 .

Numerical modeling using a commercial fluid dynamics code was used to generate results for the laminar flowfield in which the nanoparticle trajectories were calculated according to the approach described in section 2. In order to examine the influence of particle diameter on path and residence time in more than one scenario, the carotid artery geometry was scaled down to the point at which the inlet diameter would approximate an arteriole which would then branch off into capillary channels very loosely represented by the artery ICA and ECA branches. The flow conditions were chosen to approximate physiological values appropriate to the scale, as shown in table 1.

Broadly speaking, it is preferable for particles designed for drug delivery to avoid interaction with the artery walls so as not to become trapped and/or consumed by the vascular walls, which

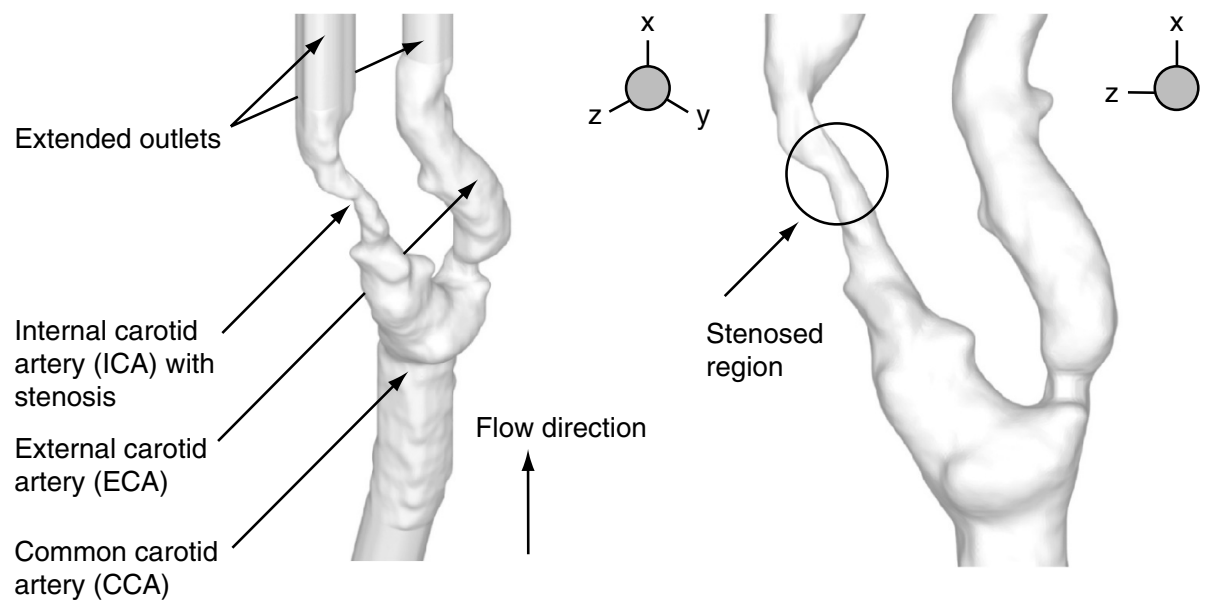

Figure 1: The carotid artery geometry. 
Table 1: Flow conditions.

\begin{tabular}{lccc}
\hline Geometry & $\begin{array}{c}\text { Mass flow in } \\
(\mathbf{k g} / \mathbf{s})\end{array}$ & $\begin{array}{c}\text { Density } \\
\left(\mathbf{k g} / \mathbf{m}^{\mathbf{3}}\right)\end{array}$ & $\begin{array}{c}\text { Dynamic Viscosity } \\
\left(\mathbf{k g} / \mathbf{m s}^{-\mathbf{1}}\right)\end{array}$ \\
\hline Artery (experimental scale) & 0.253 & 1141 & 0.008 \\
\hline Pseudo-Arteriole & $6.433 \times 10^{-10}$ & 1050 & 0.003675 \\
\hline
\end{tabular}
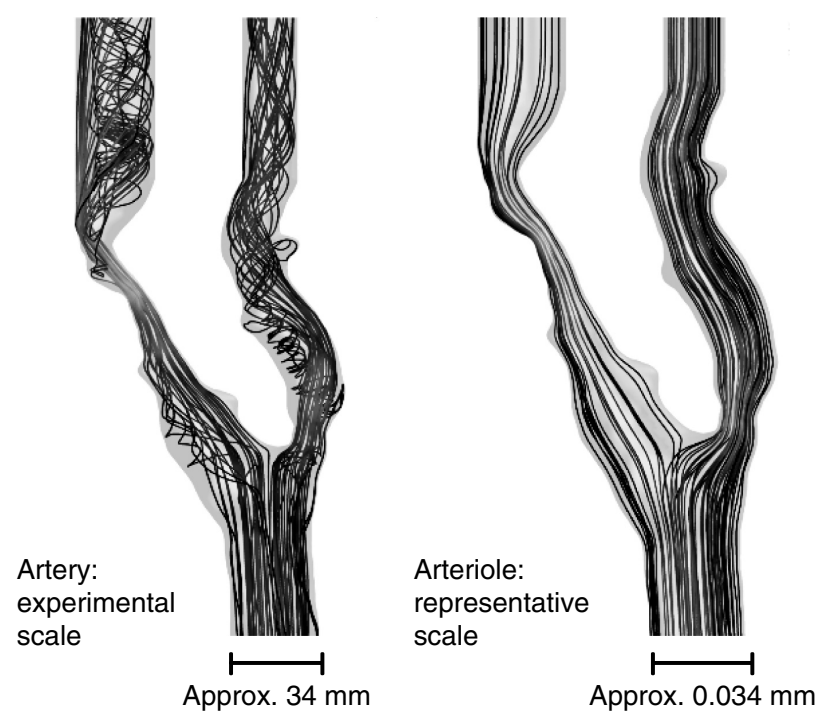

Figure 2: Flow pathlines for the artery and pseudo-arteriole.

could lead to undesirable effects. However, as the target drug delivery area will generally be attached to capillaries, enhanced propensity for interaction of particles with the capillary walls is a positive trait which will encourage penetration of, the cancerous cells requiring treatment.

The markedly different flowfields in the geometry at experiment-scale for the artery, and scaled down to that approximate to an arteriole branching into capillaries, is highlighted in figure 2 . The relatively high Reynolds number in the artery (approx. 485 based on inlet area) produces a considerable amount of swirling flow as the curvatures of the geometry induce areas of significant rotation. This is particularly clear in the ECA and following the stenosed region in the ICA, meaning that the flow has a high rotational component in the outlet channels.

Because of the greatly reduced flow rate in the small scale pseudo-arteriole, the Reynolds number is less than 1 and therefore the flow is essentially viscosity-dominated Stokes flow, with no major flow features deviating from the general path the geometry describes. The shear layers are significantly thicker than in the artery case, presenting an essentially fully-developed profile throughout the channel.

\section{METHODOLOGY}

\subsection{Numerical Method}

The commercial solver Fluent 12.1 was used to produce all the original results presented in this paper. All simulations were run in 64-bit double-precision with node-based gradient evaluation in a pressure-based segregated algorithm (SIMPLEC formulation). Various discretization schemes were evaluated, primarily to select an approach that would yield reliable steady-state results without compromising accuracy. When an attempt was made to use pure second-order upwinding for the pressure and momentum terms, the solution for the artery flow tended to fluctuate on a very small scale as a result of the solver failing to find an exact mass balance. While this may be physically-based in a small unsteadiness in the flow, the experimental data was reported as being fully steady-state, and in using a PRESTO! scheme for pressure discretization and a power law 
expression for the momentum term, a satisfactory steady-state solution was obtained with acceptable convergence.

While not typically applied to low-speed flows, the PRESTO! scheme (PREssure Staggering Option) is reported as performing well for swirling flows and flows in strongly curved domains [20], and follows the same general principles as staggered-grid schemes more commonly applied to fully-structured meshes [21]. The Power Law scheme applies a relatively straightforward linear interpolation to face values which offers a slight improvement to first-order upwinding, while acceptably damping the fluctuations present with second-order upwinding. While considerable extra investigation would be required in order to develop an approach which is more effective still, the level of flow accuracy achieved was deemed to be sufficient for the purposes of this study, as is shown in the following section.

For this preliminary study into nanoparticle transport, a simple one-way Lagrangian coupling was used, with the particle concentration deemed to be suitably low $(<1 \%)$ so as not to significantly alter the fluid properties. This approach also assumes there are no major particle-particle interactions. More importantly, the method neglects the considerable influence of red blood cells, though at the arteriole-into-capillary scale, this is a reasonable assumption as the cells generally pass single-file into the capillaries and thus a region of fluid between cells will contain only fluid and particles for a relatively large volume. The simplification of the problem further neglects thermophoretic forces and any attractive forces between particles, and wall interactions are treated as simple reflections.

As the particles are sufficiently small enough for Saffman lift and Brownian motion to become influential, the model incorporates these effects. For the purposes of this investigation, we assume that the Basset history, the virtual mass, and the Faxen correction are much smaller than the drag force acting on any isolated individual particle of this size [22]. The Brownian aspect is modeled as a Gaussian white noise after Li and Ahmadi [22].

The Brownian force components are thus of the form:

$$
F_{b_{i}}=\zeta_{i} \vee \frac{\pi S_{o}}{\Delta t}
$$

where $\zeta_{i}$ are zero-mean, unit-variance-independent Gaussian random numbers and $S_{o}$ is the spectral intensity.

The Saffman lift due to shear is incorporated based on the formulation of Li and Ahmadi:

$$
F=\frac{2 K v^{1 / 2} \rho d_{i j}}{\rho_{p} d_{p}\left(d_{l k} d_{k l}\right)^{1 / 4}}\left(v-v_{p}\right)
$$

where $K=2.594$ and $d_{i j}$ is the deformation tensor:

$$
d_{i j}=1 / 2\left(u_{i, j}+u_{j, i}\right)
$$

A basic determination of the influence of particle size was made based on predictions from the standard spherical drag of the Stokes-Cunningham formulation, which can be expressed as:

$$
F_{D}=\frac{18 \mu}{d_{p}^{2} \rho_{p} C_{c}}
$$

where $C_{c}$ is the Cunningham correction based on the molecular mean free path [23] - this is effectively 1 in the present case due to the relatively high density of the fluid. 
For simulations involving particle Brownian motion, 100 separate particle release operations were conducted, with each of these operations involving the passive injection of approximately 4000 particles into the fluid from a plane close to the inlet. From the computed particle track statistics, means for minimum, maximum and mean particle residence time for particles travelling through the ICA and ECA were calculated. A standard deviation was deduced from this data and the addition of a further 100 tracking operations altered this deviation by less than $1 \%$ and the means by a negligible amount, thus showing that the original 100 operations were sufficient to gather reliable statistical data. With approximately 4000 particles tracked in each instance, the mean residence times were therefore based on around $4 \times 10^{5}$ individual particle paths.

The following sections establish the effectiveness of the numerical method in reproducing the reference experimental results [19], and present flowfields from simulations scaled to actual-artery and capillary size. Features of nanoparticle transport through these geometries are then discussed.

Extensive testing revealed that the numerical flowfield was borderline unsteady, given the large number of recirculating regions and areas of strong velocity gradients, and therefore quite mesh-sensitive. A true second-order steady state solution was possible given considerable under-relaxation of the pressure and momentum variables on coarse and medium meshes at the start of the solution process, but the fine mesh retained some very minor flow instability. However, in all cases, an overall better match to experimental results with stable convergence was produced using a PRESTO! (Pressure Staggering Option) discretization for the pressure term and a power law spatial discretization scheme. All results quoted in the following sections were produced in this manner.

\subsection{Validation}

The experiments of Cheung et al. form the basis of the validation for the present study, as the geometry used here is identical apart from some extension to the inlet and outlet to aid with numerical stability at the inlet and outlet. The high level of rotation seen in the outlet channels in figure 2 was the most likely reason for difficult convergence with a shorter outlet region. As the flow was essentially fully-developed upon arrival at the MRI-derived geometry in the experiment, this alteration was not influential to the comparison flowfield, and the additional computational expense of the extended inlet and outlet was justified by improvements to convergence.

The flow is assumed to be laminar at all points, although it is likely that transition occurred at the stenosis, where the local Reynolds number is well in excess of 2000. Experimental data in this region was not reliable, however, and for the purposes of the present investigation the transition is not an important feature, as particle transport in turbulent arterial flows is worthy of its own investigation.

Numerical results reported in the reference paper ([19]) were produced using a relatively similar approach to that adopted for the present study. In that instance, the Fluent code was used featuring meshes with a maximum of $1.52 \times 10^{6}$ cells in the finest mesh, which was stated as producing "grid-independent" results due to a difference of less than $0.2 \%$ in maximum velocity through the stenosed region compared to that predicted by a mesh of $0.29 \times 10^{6}$ cells. Second-order upwinding was used to produce those results, but it should be noted that the near wall region did not feature any special mesh treatment to resolve the boundary layer in detail as it does in the present study. The numerical match to experimental results was mixed, with a strong tendency to over-prediction of axial velocities, particularly in the ECA, of margins as high as $30-55 \%$ in some places. The overall trends were reasonably well predicted at most reference points, however.

Coarse, medium and fine meshes were constructed for the present study using a hybrid approach clustering anisotropic prism mesh in the boundary layer region and utilizing relatively uniform-size tetrahedral cells in the remaining body of the volume. Some more concentrated local refinement was introduced at the bifurcation and the stenosed region to better resolve the strong gradients there, as seen in figure 3 , which depicts a general overview of the standard mesh.

The meshes were not refined linearly, but rather incrementally in terms of surface and volume cell size. The mesh spacing growing tangential to the wall was kept relatively constant, as it was appreciated that particle/wall interactions would be particularly sensitive to the resolution in this region and it would have to be relatively fine whatever the cell density elsewhere. The coarse mesh contained a total of $3.31 \times 10^{6}$ cells, the standard mesh a total of $5.36 \times 10^{6}$, and the fine mesh a total of $10.32 \times 10^{6}$ cells. 


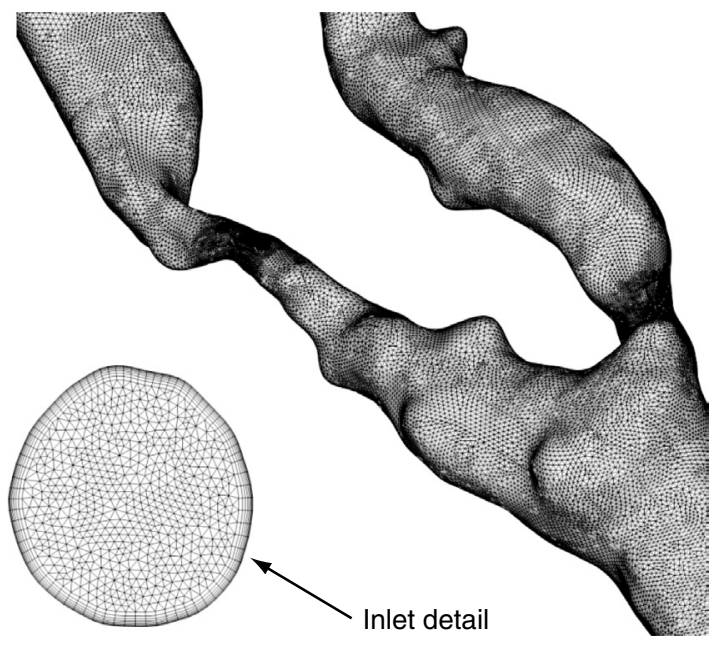

Figure 3: The standard mesh, $5.36 \times 10^{6}$ volume cells.

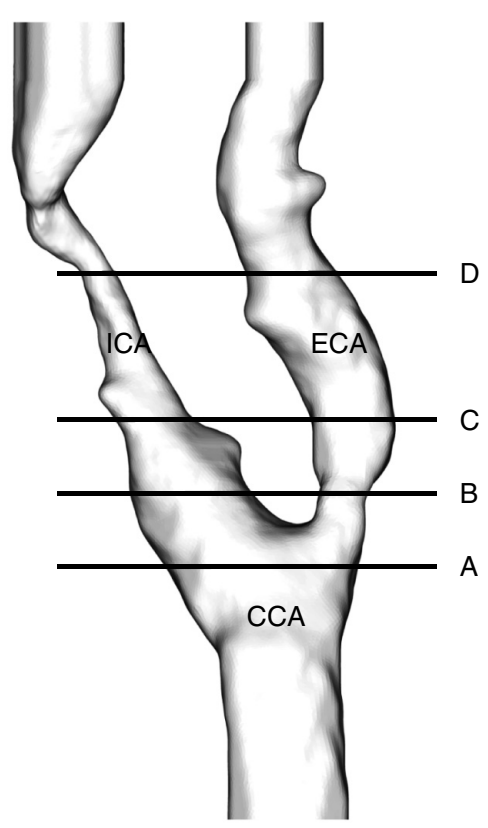

Figure 4: Locations for numerical comparison to experimental velocity profiles.

Figure 4 depicts the artery geometry showing locations of cuts where streamwise velocity profiles were extracted, on a plane which intersects the majority of the domain. These cuts match those reported in literature, though we note here that the plane location at cut D exists closer to the artery wall than the thickness of the laser light sheet $(2 \mathrm{~mm})$, and therefore the experimental data in this location is not treated as reliable and is ignored in the present study.

It is clear from the results shown in fig. 5 (particularly in the boundary layer at cut c) in the ECA) that true mesh-independence has not been achieved with the fine mesh, even with close to an order of magnitude more cells than the finest mesh reported in the original comparison in literature [19]. However, improvements on that study have been achieved, with the majority of comparison points offering improved agreement with the experiments. There are still significant discrepancies, however, with the mis-match between numerical and experimental data (featuring both over- and under-prediction at points) in the CCA at comparison A having a knock-on effect for the rest of the flowfield downstream. At this initial stage, all three meshes predict very similar velocity profiles, with the fine mesh marginally closer to the peak velocity. At comparison point $\mathrm{B}$, following the 


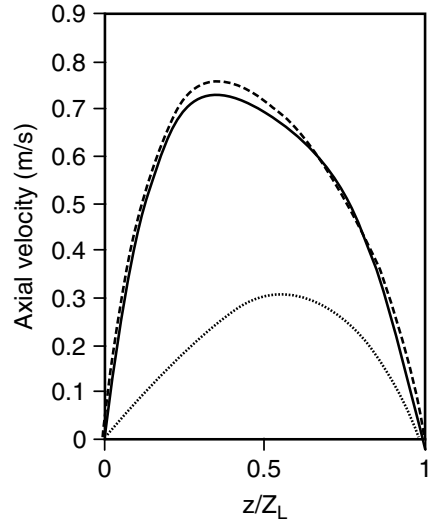

(d) ICA

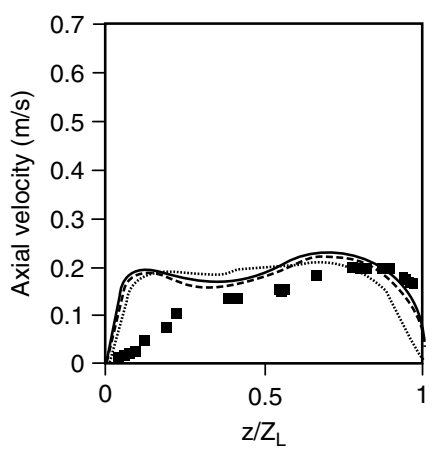

(c) ICA

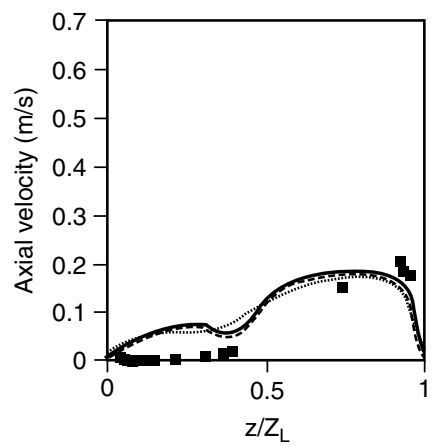

(b) ICA

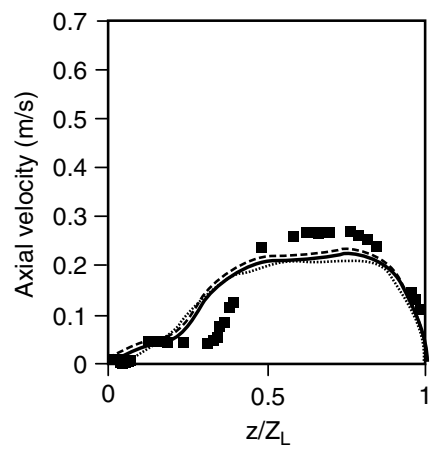

(a) CCA

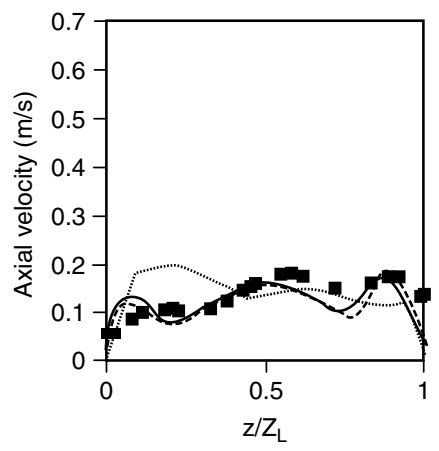

(d) ECA

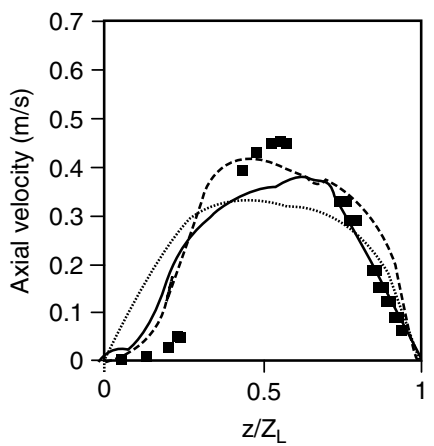

(c) ECA

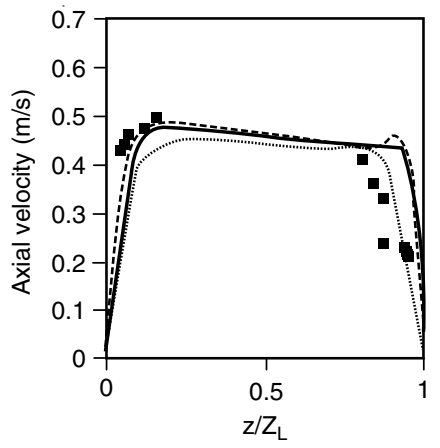

(b) ECA

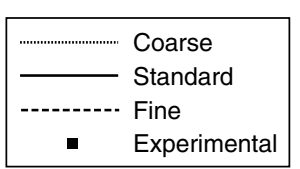

Figure 5: Velocity profiles compared to experimental results for coarse, standard and fine meshes, taken at the locations indicated in figure 4.

bifurcation of the artery, the three meshes again offer similar profiles in the ICA but the coarse mesh under-predicts the peak velocities in the ECA by a wider margin. This trend is repeated in the ECA at comparison $\mathrm{C}$, where the three meshes produce markedly different profiles and only the fine 
mesh is close to describing the peak velocities there. In the ICA at cut C, all meshes greatly overestimate the velocity profile from $z / Z_{L}=0$ to at least 0.4 , with a closer match over the remaining portion, possibly as an exaggeration of the trend which began at cut $\mathrm{B}$. At cut $\mathrm{D}$ the standard and fine meshes predict the ECA profile relatively well considering earlier discrepancies, but the coarse mesh has a velocity bias towards the wall at $z / Z_{L}=0$ that produces a poor match up to $z / Z_{L}=0.3$. In the ICA, the coarse mesh is clearly inadequate for resolving the velocity profile. With a very narrow channel and very few mesh points to describe the flowfield, the lowest-velocity portions of the shear layers are exaggerated and merge causing a relatively low velocity at the point of maximum constriction, whereas the higher resolution of the standard and fine meshes indicate a considerably higher velocity peak.

Overall, while the trends are generally well repeated with the standard and fine meshes, with the fine mesh offering the preferred comparison to the experiment, there are areas where the numerical results offer relatively poor correlation. In perspective, however, the present modeling offers an improvement over that reported in literature, and indeed it must be noted that the experimental results do not quote any reliable measure of error despite some of the measurements being obtained in the boundary layers close to the walls (particularly at ECA c) and ICA d)). For the purposes of examining nanoparticle transport, the comparisons were deemed to be satisfactory with the fine mesh, and thus all results reported in the following section were created using this approach.

The pseudo-arteriole, as already mentioned, offered a very different flow regime from that found in the artery. Comparison experiments were not available for this Reynolds number, and the three meshes produced near-identical results due to the much larger shear layers and lack of vertical flow behaviour. The standard mesh was used in this instance as the flowfield is markedly more simplistic than in the artery case, featuring no recirculation zones or indeed any significant rotational behaviour. Velocity profiles, as with the artery but at the smaller scale, are presented in figure 6 for reference and to highlight the different flow behaviour as compared qualitatively to the artery, including the lack of any major velocity peak at the "stenosed" region.

(a)

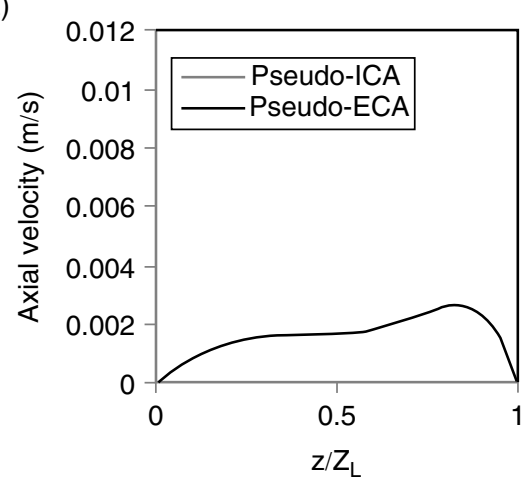

(c)

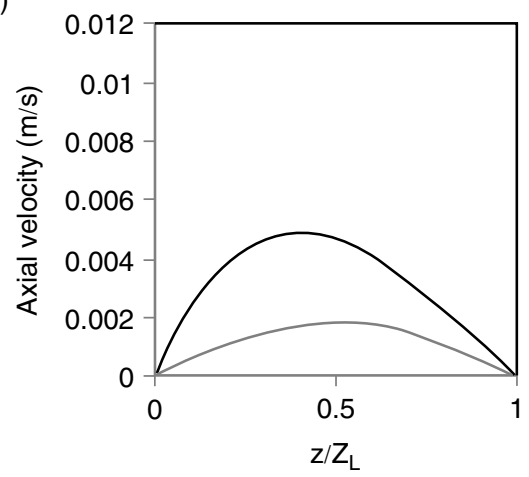

(b)

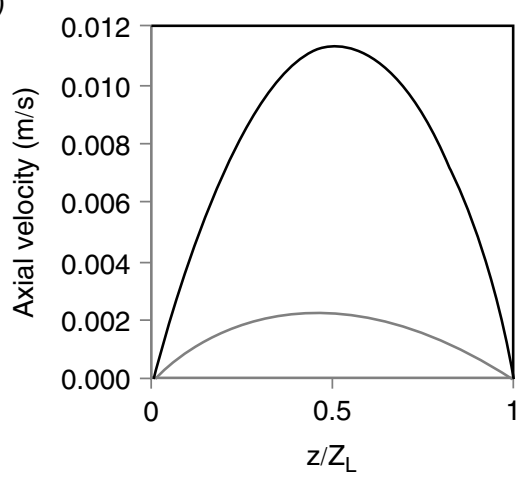

(d)

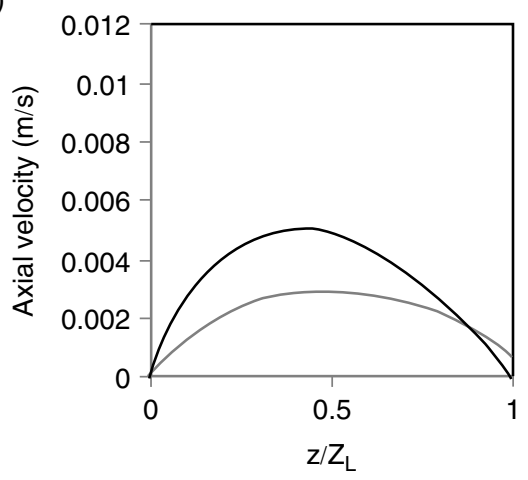

Figure 6: Velocity profiles for pseudo-arteriole at equivalent positions to those shown in fig. 4. 


\section{RESULTS}

\subsection{Artery, Experiment Scale}

The results presented are focused around particle residence time, which is the total time spent in the geometry from inlet to outlet. This parameter is of interest to designers of nanoparticles for drug delivery as it not only determines how swiftly the particles will navigate the vasculature to arrive at the site of interest, but can determine how long particles will stay in the entire bloodstream if circulating throughout the body and therefore may determine the extent of any toxic side-effects.

Figure 7 indicates that the average residence time for the particles in the artery reduces with a corresponding reduction in particle diameter, with total residence time for a $50 \mathrm{~nm}$ particle being around $13 \%$ less than that for the $500 \mathrm{~nm}$ particle in the ICA and $9 \%$ less in the ECA. Clearly the region of highly-accelerated flow through the stenosis in the ICA reduces the average residence time for all particles compared to the passage through the ECA, but the reduced drag of the smaller particles means that the effect is more pronounced in the ICA.

These trends are not surprising, but these overall mean values mask some more pronounced effects. Looking at the mean values (for all 100 trajectory tracking operations) for maximum and minimum particle residence time, as non-dimensionalised against the overall mean of all particles over all trajectory tracking operations, some more interesting trends can be observed in figure 8 .

While minimum particle residence time in both branches stays the same in absolute terms, the reducing mean residence times results in the minimum residence time increasing relative to the mean with reducing particle diameter, by approximately $9 \%$ in the ICA. Conversely, the maximum residence time decreases markedly (in absolute and non-dimensionalised frames of reference) from $500 \mathrm{~nm}$ particles to $300 \mathrm{~nm}$ particles (by close to $50 \%$ ). Similar characteristics are observed for the particles travelling through the ECA, where the reduction in maximum residence time is not as pronounced as in the ICA, particularly when comparing the change from $500 \mathrm{~nm}$ to $300 \mathrm{~nm}$ and from $300 \mathrm{~nm}$ to $100 \mathrm{~nm}$, but from $500 \mathrm{~nm}$ to $50 \mathrm{~nm}$ the $t_{\text {max }} / t_{\text {mean }}$ drops by close to $45 \%$.

Several factors are behind these trends. Firstly, as figure 9 indicates, the number of particles experiencing one or more wall interactions significantly increases as the particle diameter is reduced. The percentage essentially doubles when comparing $500 \mathrm{~nm}$ to $50 \mathrm{~nm}$ particles. While the current model treats these interactions as simplistic reflections, it is clear that this produces conservative results as particle/wall interactions in actual vasculature would be more complex and potentially trap a large percentage of these interacting particles - precisely the trait which makes

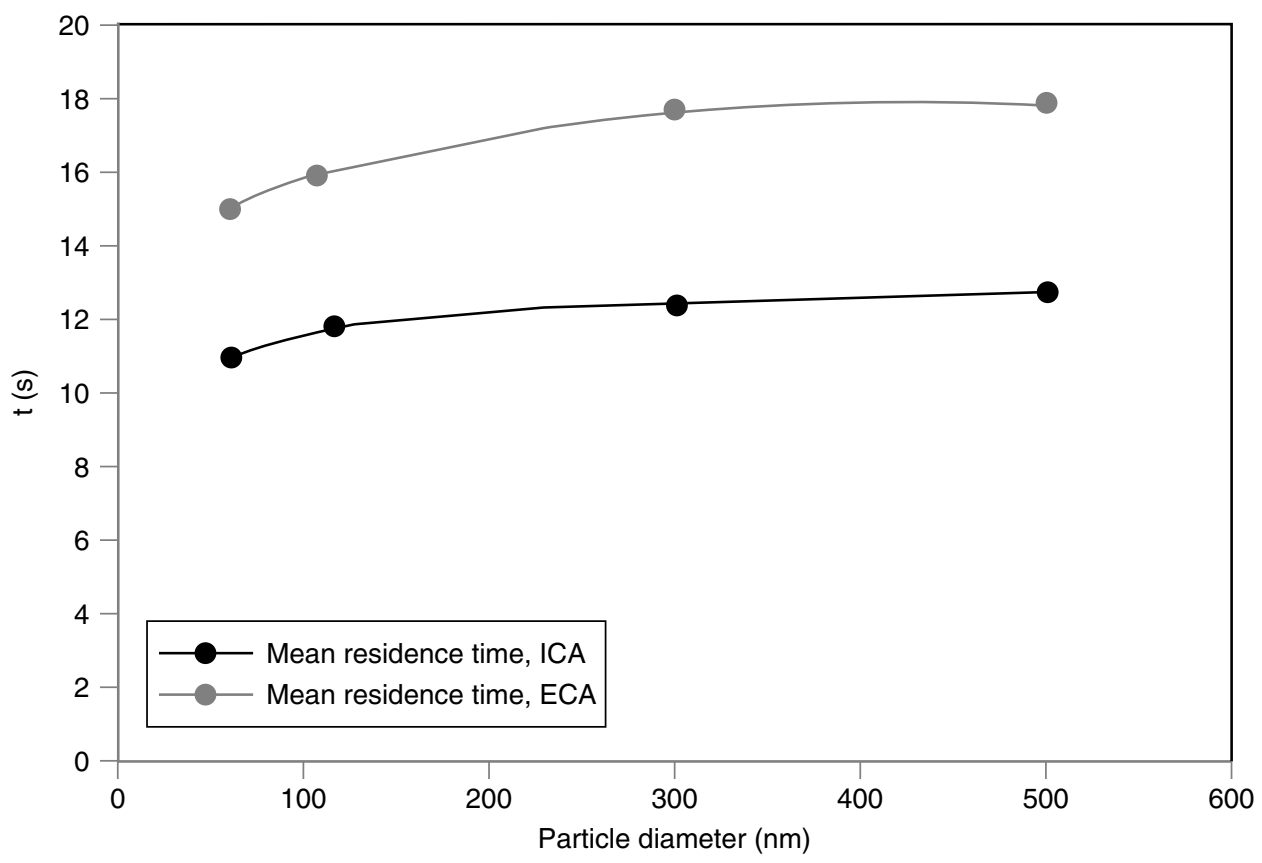

Figure 7: Mean particle residence time (absolute) vs. particle diameter in the artery (experiment scale). 


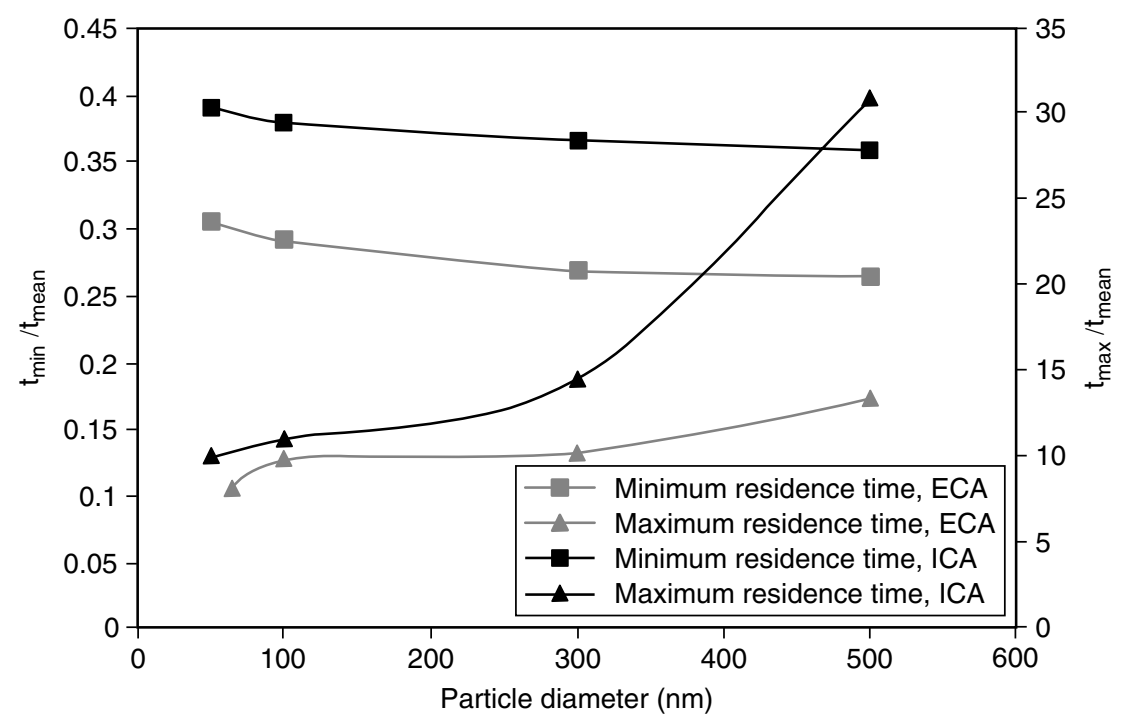

Figure 8: Minimum $\left(t_{\min }\right)$ and maximum $\left(t_{\max }\right)$ particle residence times nondimensionalised against the mean vs. particle diameter.

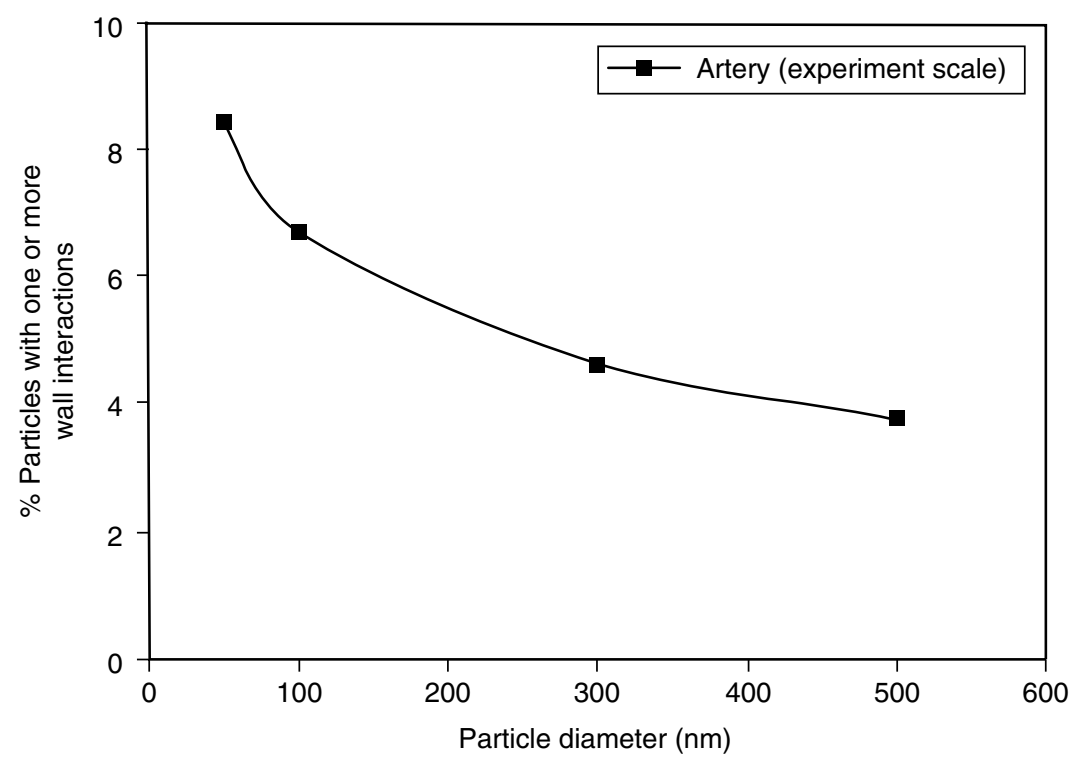

Figure 9: Percentage of particles experiencing one or more wall interactions vs. particle diameter.

smaller particles desirable to researchers looking to target tumours in certain organs. The increase in wall interactions is due to the Brownian influence on particle motion; smaller particles are more highly influenced due to the larger Brownian force moving them across streamlines into the slowmoving fluid next to the walls. Here, Brownian diffusion is more pronounced than the advective transport of particles travelling in the fast-moving fluid in the middle of the volume.

For particles which are able to move away from the walls from the lift due to shear, the lack of further wall interactions plus the move to faster moving flow in the middle of the channel combines with the reduced drag of the smaller particles to greatly reduce the maximum residence time. The enhanced influence of the Saffman lift on small particles is evident in this respect, though the increased number of wall interactions indicates that the effect is gradual.

To better understand the role Brownian motion plays in influencing the trajectories of particles of differing sizes, the results in figure 10a for the ICA and figure 10b for the ECA are presented in 
(a)

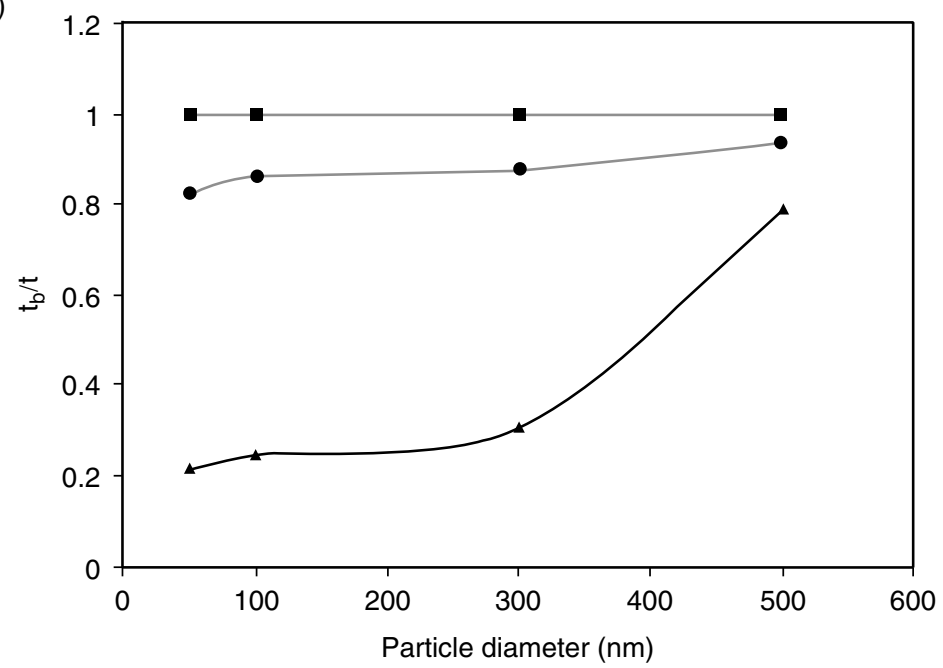

(b)

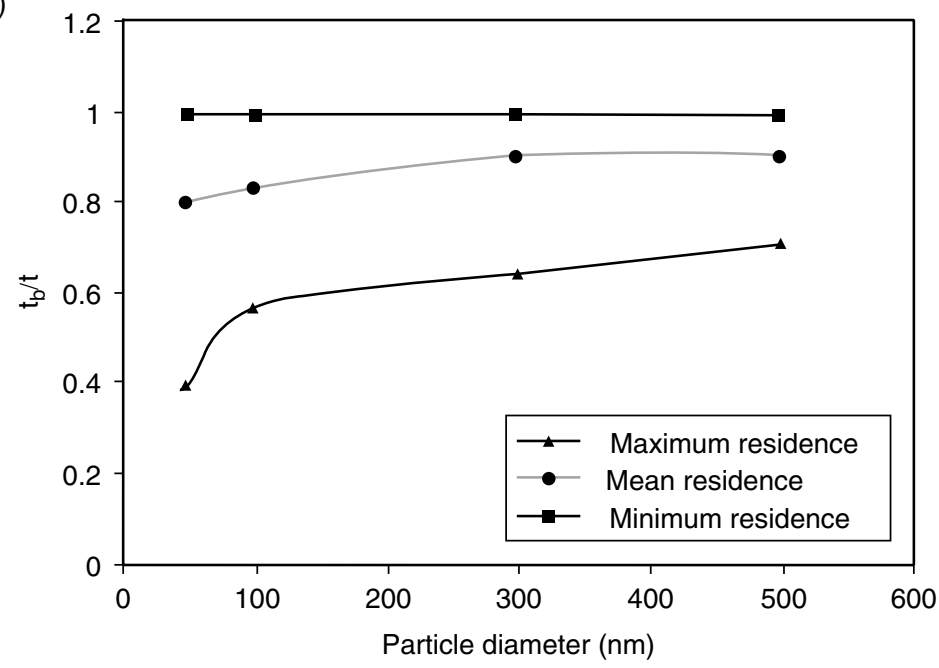

Figure 10: Max., min. and mean residence times for particles with Brownian motion $\left(t_{b}\right)$, non-dimensionalised against values from non-Brownian particles $(t)$, vs. particle diameter for a) ICA and b) ECA branches.

terms of maximum and minimum particle residence time as non-dimensionalised against the comparison residence time for particle paths without Brownian motion. From these graphs it is clear to see that the minimum residence time is negligibly affected by Brownian motion; as stated previously, the flow is moving too quickly to allow the Brownian influence to change the particle trajectory by any meaningful degree, and the particles are too small to suffer any meaningful retardation in the high-velocity regions.

The mean residence time for the cases with Brownian motion is actually reduced compared to cases without, and indeed decreases by a greater margin with reducing particle diameter, which initially seems counter-intuitive. However, this may be explained in part by the wall interactions which occur in the Brownian cases but not in the reference cases. The particles involved in such interactions will tend to rebound into the fluid with energy which will enhance their ability to be lifted, even slightly, away from the slowest flow next to the wall and into the faster-moving fluid closer to the middle of the channel. This compares to the non-Brownian case, where the particles can remain trapped close to the wall for the duration of their time in the artery.

The increased flow acceleration in the stenosis of the ICA exaggerates the trend for particles in that branch, as the accelerated particles are less likely to marginate again once passing through the narrowest section. In these simulations there is a therefore general reduction in maximum residence 
time, however in a real situation a potentially-greater proportion of the particles wouldbecome trapped at the wall and thus be removed from circulation within the body - generally an undesirable outcome for an artery, but a positive trait for particles in slow-moving fluid in the vicinity of cancerous cells.

As an aside, the results indicate that the lower limit for size of seeding particles for experimental fluid-only work on geometries such as the artery, involving laser-measurement approaches such as PIV and LDA, must be considerably higher than $500 \mathrm{~nm}$ if the results are to be accurate and free of undue wall interactions and excessive influence from Brownian motion and particle lift due to the shear flow close to the boundaries.

\subsection{Pseudo-Arteriole}

With the geometry scaled down markedly from the artery case (preserving the relative mesh as identical) such that the region of maximum constriction is just 6 microns, the trends observed in the previous section do not necessarily hold in the flow conditions of the pseudo-arteriole. With the Reynolds number less than one for flow entering the bifurcated channels, which we will refer to as capillaries in the spirit of the scale, the Saffman lift due to shear is not particularly influential, but the Brownian diffusion of the particles is considerable in the low-velocity flowfield. The latter characteristic is reflected in figure 11, which highlights a slight increase in mean residence time as particle diameter is reduced from $500 \mathrm{~nm}$ to $100 \mathrm{~nm}$, and a more marked increase from $100 \mathrm{~nm}$ to $50 \mathrm{~nm}$. The Peclet number is approximately three orders of magnitude less than for the artery, indicating a much higher influence of particle diffusion relative to advection.

Unlike the artery case, where minimum residence time was unaffected by changes to particle diameter, the minimum residence times in the pseudo-arteriole case decrease slightly in absolute terms. The maximum residence time reduces by a more significant percentage with increases in particle diameter. Non-dimensionalising these parameters against the mean as before, in figure 12 , indicates that the maximum residence in the "ECA" branch increases with reducing particle diameter from $500 \mathrm{~nm}$ to $100 \mathrm{~nm}$, but then reduces sharply from $100 \mathrm{~nm}$ to $50 \mathrm{~nm}$, while in the "ICA" branch the maximum residence time decreases consistently as the particle diameter shrinks. In both branches of the geometry, the minimum residence time reduces.

Figure 13 highlights the percentage of particles involved in one or more wall interactions, and although the trend of increased interaction with decreased particle diameter seen in the artery case holds, the percentages are much higher, with over $33 \%$ of all $50 \mathrm{~nm}$ particles meeting the wall at some point. This compares to just over $18 \%$ for $500 \mathrm{~nm}$ particles. Clearly the Brownian influence is inducing these interactions in a much more pronounced fashion than at the larger scale and higher Reynolds number.

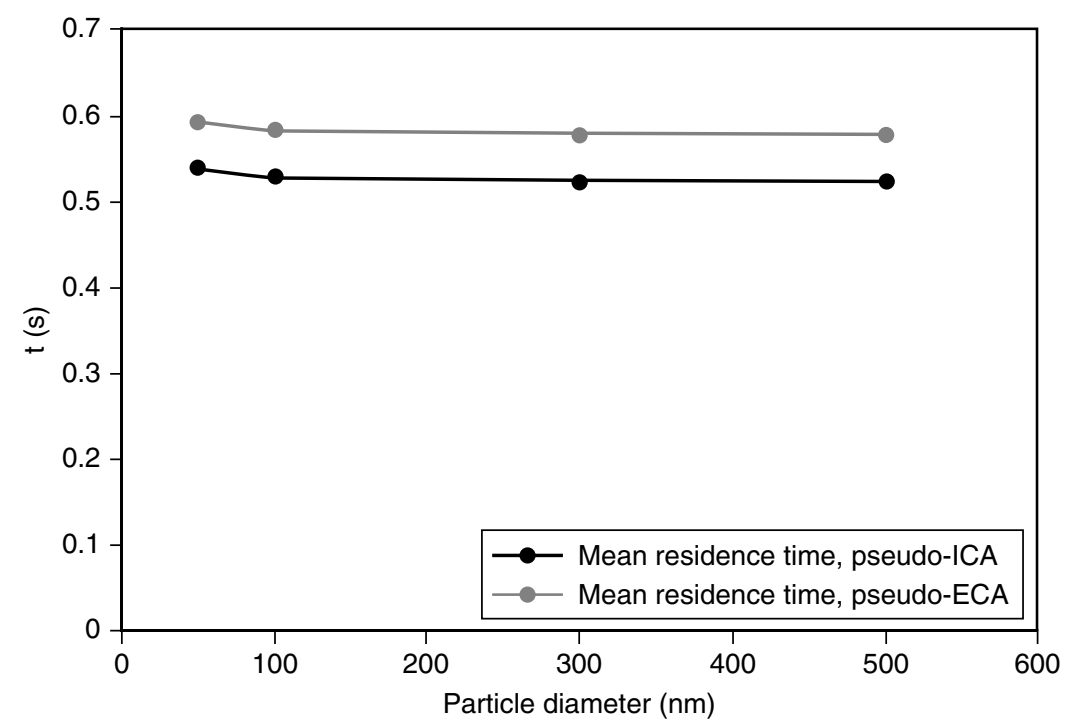

Figure 11: Mean particle residence time (absolute) vs. particle diameter. 


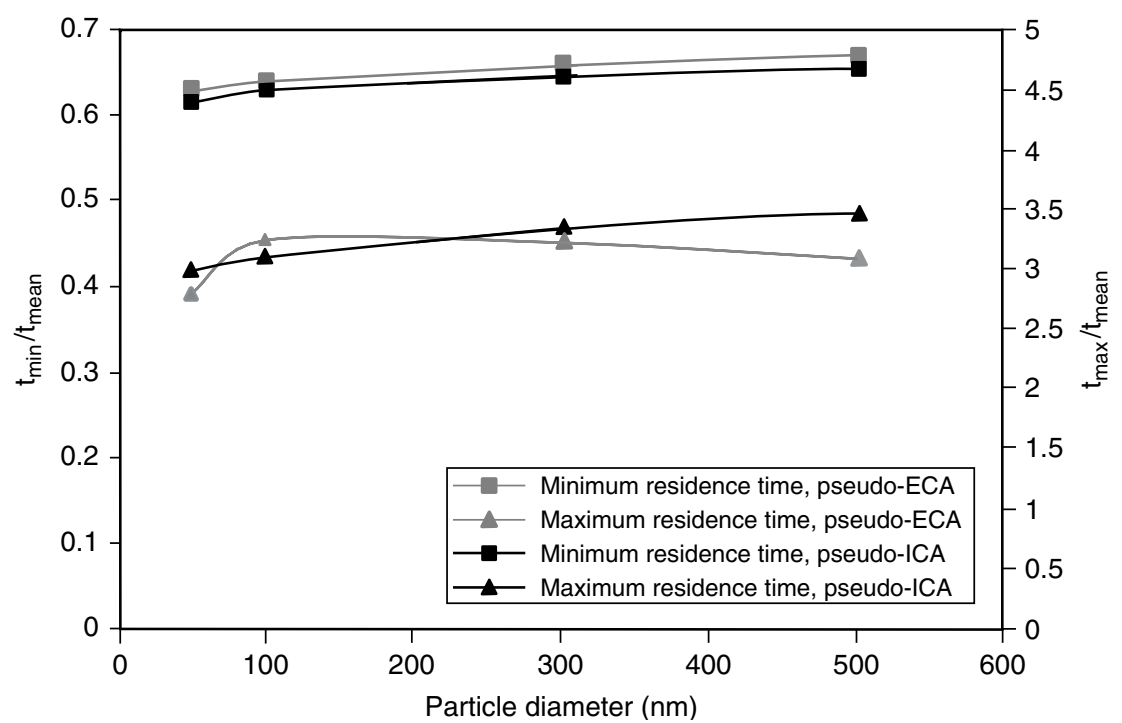

Figure 12: Minimum $\left(t_{\min }\right)$ and maximum $\left(t_{\max }\right)$ particle residence times, nondimensionalised against the mean $\left(t_{\text {mean }}\right)$, vs. particle diameter.

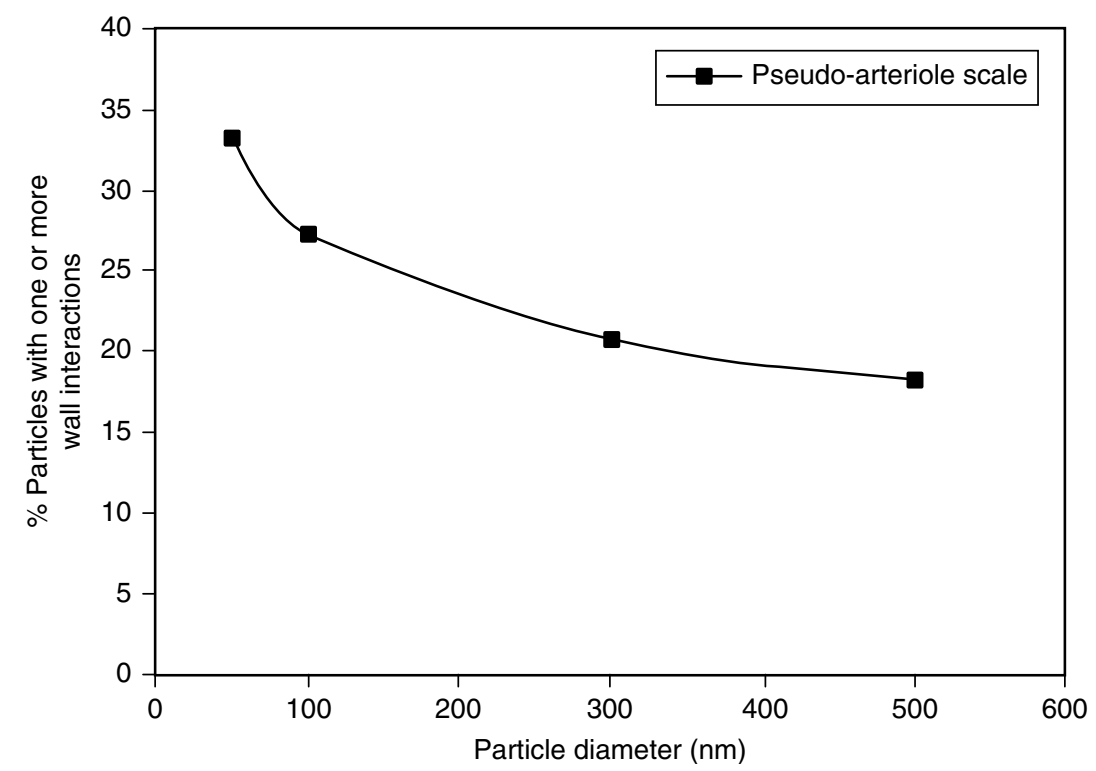

Figure 13: Percentage of particles experiencing one or more wall interactions vs. particle diameter.

With particles of smaller diameter, the relative disturbance to particle trajectory due to this effect is stronger and thus particles closer to the wall are far more likely to have the Brownian force overcome other forces acting on it to keep it from the boundary.

Characterising the strong influence of Brownian motion on the particles compared to those in cases run without this effect, in figure 14a for the "ICA" and figure 14b for the "ECA", one can see that the maximum residence time for the Brownian cases is markedly higher, undoubtedly due to the increased length of the overall particle trajectories due to the additional deviations. As particle diameter reduces from $500 \mathrm{~nm}$ to $50 \mathrm{~nm}$, so too does the relative maximum residence time, though it does not approach the non-Brownian level. In the "ECA" case, as also noted for figure 12, the maximum residence actually increases with reducing particle diameter from $500 \mathrm{~nm}$ to $100 \mathrm{~nm}$, before the overall trend is restored at $50 \mathrm{~nm}$.

The minimum residence time in both channels reduces by a small margin with Brownian motion compared to the particles without; most likely a function of the wall reflections, as with the artery, 

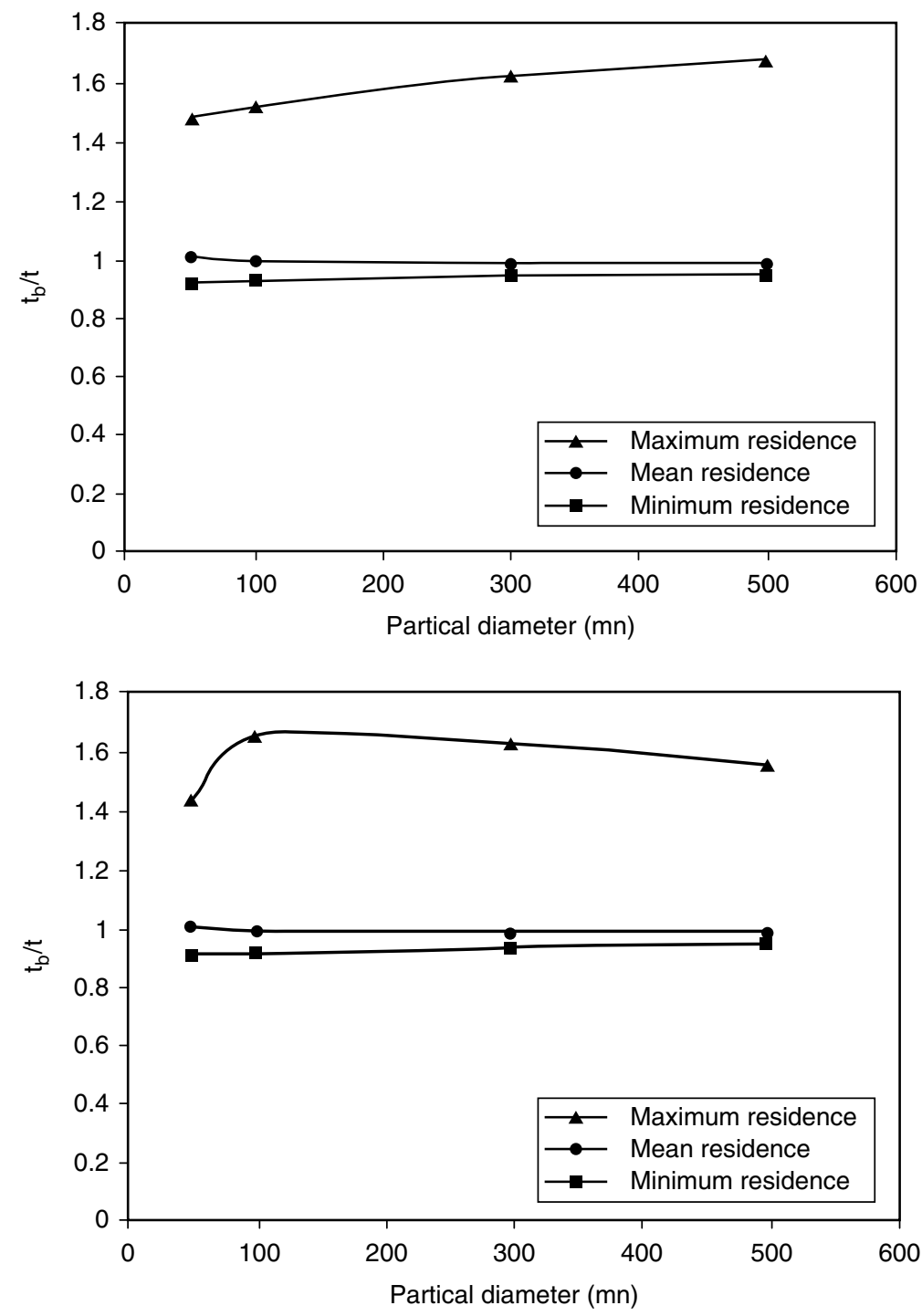

Figure 14: Max., min. and mean residence times for particles with Brownian motion $\left(t_{b}\right)$, non-dimensionalised against values from non-Brownian particles $(t)$, vs. particle diameter for a) ICA and b) ECA branches.

inducing the particles to move back into the shear layer rather than hold station in the slowestmoving fluid near the wall.

\section{CONCLUSIONS}

A preliminary numerical study into nanoparticle transport in a bifurcated carotid artery, and a scaled-down version approximating an arteriole and branch capillaries, has been conducted to establish the influence of size on the trajectory and residence time of particles. Diameters of $500 \mathrm{~nm}, 300 \mathrm{~nm}, 100 \mathrm{~nm}$ and $50 \mathrm{~nm}$ were investigated using a simple one-way fluid coupling. In the case of the artery, a decrease in particle size was accompanied by a decrease in mean particle residence time. The minimum residence time was generally unaffected but the maximum residence time dropped sharply with reducing particle size, primarily because of the lower drag and considerably higher influence of Saffman lift on the smaller. The percentage of particles experiencing wall interactions effectively doubled with particle diameter reducing from $500 \mathrm{~nm}$ to $50 \mathrm{~nm}$ to $8.4 \%$.

In the pseudo-arteriole, where the conditions are essentially those of Stokes flow, the mean residence time increases by approximately $3 \%$ with reducing particle diameter from $500 \mathrm{~nm}$ to 
$50 \mathrm{~nm}$, primarily due to the increased influence of Brownian motion as the mass is reduced. However, both the minimum and maximum residence times decreased. At $500 \mathrm{~nm}, 18 \%$ of particles experienced wall interactions, whereas this figure for the $50 \mathrm{~nm}$ particles was closer to $33 \%$. Again, this highlights the significant influence of Brownian motion on the smallest particles closest to the walls.

This initial study highlights the importance of particle size for the efficacy of nanoparticle drug delivery, and the work is currently being extended to a transient flow imitating systolic pulsatile flow, where Brownian diffusion plays a much stronger role even in the artery during stages where the peak flow velocity is close to zero. Methods of four-way coupling are being implemented which will provide a means by which to incorporate particle attraction forces and more realistic agglomeration effects and accretion at the wall.

\section{ACKNOWLEDGEMENTS}

We wish to thank Professor Jiyuan Tu at the School of Aerospace, Mechanical \& Manufacturing Engineering, and Health Innovations Research Institute (HIRi) for his involvement in providing the artery geometry and access to experimental results.

\section{REFERENCES}

[1] Peer, D., Karp, J.M., Hong, S., Farokhzad, O.C., Margalit, R., Langer, R., "Nanocarriers as an emerging platform for cancer therapy", Nature Nanotechnology, 2, 2007.

[2] Decuzzi, P., Godin, B., Tanaka, T., Lee, S.Y., Chiappini, C., Liu, X., Ferrari, M., "Size and shape effects in the biodistribution of intravascularly injected particles", Journal of Controlled Release, 141, 2010.

[3] Johnston, H.K., Hutchison, G., Christensen, F.M., Peters, S., Hankin, S., Stone, V., "A review of the in vivo and in vitro toxicity of silver and gold particulates: Particle attributes and biological mechanisms responsible for the observed toxicity”, Critical Reviews in Toxicology, 40, (4), 2010, pp. 328-346.

[4] Ferrari, M., "Cancer nanotechnology", Nat. Rev. Cancer, 5, 2005.

[5] Gaumet, M., Vargas, A., Gurny, R., Delie, F., "Nanoparticles for drug delivery: The need for precision in reporting particle size parameters". European Journal of Pharmaceutics, 69, pp. 1-9.

[6] Ruenraroengsak, P., Cook, J.M., Florence, A.T., "Nanosystem drug targeting: facing up to complex realities", Journal of Controlled Release, 141, 3, 2010.

[7] Champion, J., Katare, Y.K., Mitragotri, S., "Particle shape: a new design parameter for micro- and nanoscale drug delivery carriers", Journal of Controlled Release, 121, 2007.

[8] Beningo, K.A., Wang, Y., "Fc-receptor-mediated phagocytosis is regulated by mechanical properties of the target", Journal of Cell Science, 115, 2002.

[9] Nakaoka, R., Tabata, T., Yamaoka, T., Ikada, Y., "Prolongation of the serum half-life period of superoxide dismutase by poly(ethylene glycol) modification”, Journal of Controlled Release, 46, (1997), pp. 253-261.

[10] Moghimi, S.M., Hunter, A.C., Murray, J.C., "Long-circulating and target-specific nanoparticles: theory to practice", Pharmacol. Rev., 53, (2001), pp. 283-318.

[11] Moghimi, S.M., "Mechanisms of splenic clearance of blood cells and particles: towards development of new splenotropic agents", Adv. Drug Deliv. Rev., 17, (1995), pp. 103-115.

[12] Moghimi, S.M., "Exploiting bone marrow microvascular structure for drug delivery and future therapies", Adv. Drug Deliv. Rev., 17, (1995), pp. 61-73.

[13] Banerjee, T., Mitra, S., Kumar, S.A., Kumar, S.R., Maitra, A., "Preparation, characterization and biodistribution of ultrafine chitosan nanoparticles", Int. J. Pharm., 243, (2002), 93-105.

[14] Storm, G., Belliot, S.O., Daemen, T., Lasic, D.D., "Surface modification of nanoparticles to oppose uptake by the mononuclear phagocyte system”, Adv. Drug Deliv. Rev., 17, (1995), pp. 31-48.

[15] Doshi, N., Prabhakarpandian, B., Rea-Ramsey, A., Pant, K., Sundaram, S., Mitragotri, S., "Flow and adhesion of drug carriers in blood vessels depend on their shape: A study using model synthetic microvascular networks", Journal of Controlled Release, 146, 2, 2010.

[16] Decuzzi, P., Pasqualini, R., Arap, W., Ferrari, M., "Intravascular delivery of particulate systems: does geometry really matter?", Pharmaceutical Research, 26 (1), 2009.

[17] Fahraeus, R., Lindqvist, T., "The viscosity of the blood in narrow capillary tubes", American Journal of Physiology, 96, 1931. 
[18] Charoenphol, P., Huang, R.B., Eniola-Adefeso, O., "Potential role of size and hemodynamics in the efficacy of vascular-targeted spherical drug carriers", Biomaterials, 31 (6), 2010.

[19] Cheung, S.C.P., Wing, K.K.L., Yeoh, G.H., Yang, W., Tu, J., Beare, R., Phan, T., "Experimental and numerical study on the hemodynamics of stenosed carotid bifurcation", Australasian Physical and Engineering Science in Medicine, 33 (4), pp. 319-328.

[20] Fluent Inc. "Fluent 6/.3 User's Guide, Vol. 2", Lebanon, NH, 2006.

[21] Patankar, S.V. Numerical Heat Transfer and Fluid Flow, Hemisphere, Washington DC, 1980.

[22] Li, A., Ahmadi, G., "Dispersion and Deposition of Spherical Particles from Point Sources in a Turbulent Channel Flow", Aerosol Science and Technology, 16, pp. 209-226, 1992.

[23] Cunningham, E., "On the velocity of steady fall of spherical particles through fluid medium," Proc. Roy. Soc. A 83 (1910) 357. 
